\title{
Pathotropic targeting advances clinical oncology: Tumor-targeted localization of therapeutic gene delivery
}

\author{
FREDERICK L. HALL ${ }^{1}$, JOHN P. LEVY ${ }^{1}$, REBECCA A. REED ${ }^{1}$, WASINEE N. PETCHPUD ${ }^{1}$, \\ VICTORIA S. CHUA ${ }^{2}$, SANT P. CHAWLA ${ }^{2}$ and ERLINDA M. GORDON ${ }^{1}$ \\ ${ }^{1}$ Oncology Research Unit, Epeius Biotechnologies Corporation, San Marino CA 91108, \\ and ${ }^{2}$ Sarcoma Oncology Center, 2811 Santa Monica Boulevard, Suite 414, Santa Monica, CA 90403, USA
}

Received June 23, 2010; Accepted July 22, 2010

DOI: 10.3892/or_00000926

\begin{abstract}
The advent of pathotropic (disease-seeking) targeting has transported genetic medicine across the threshold of history with the progressive clinical validation of Rexin-G, a tumor-targeted nanosized anti-cancer agent. Achieving noteworthy single-agent efficacy and survival benefits in otherwise intractable cancers, the molecular biotechnology platform has stimulated intense interest in the underlying mechanisms-of-action. This report exhibits the effective localization of Rexin-G nanoparticles within a metastatic liver lesion, as observed upon its surgical excision.
\end{abstract}

\section{Introduction}

Rexin-G is a pathotropically-targeted replication-incompetent retroviral vector encoding a dominant-negative mutant form of the human cyclin G1 gene, an essential component of the executive cell cycle control pathways that are fundamental to cell growth $(1,2)$. By targeting and thus aborting this critical regulatory component of the cell's universal replication machinery, Rexin-G is invariably lethal to cancer cells derived from all three germ layers, including their associated proliferative vasculature, which empowers Rexin-G with potent anti-angiogenic properties $(3,4)$, as well as broad spectrum tumoricidal activity (5-7). Each therapeutic nanoparticle incorporates a highly efficient and exquisitely specific targeting function to seek out and accumulate in cancerous tissues, using the abnormal or 'pathological' properties of the disease itself, specifically, the newly exposed collagenous proteins associated with cancer growth, metastasis, and tumor-associated blood vessel

Correspondence to: Dr Erlinda M. Gordon, Oncology Research Unit, Epeius Biotechnologies Corporation, San Marino CA 91108, USA

E-mail: emgordon@epeiusbiotech.com

Key words: targeted gene delivery, pathrotropic nanoparticles, nanomedicine, cell cycle control, cyclin G1, apoptosis formation (8). The first targeted genetic medicine of its kind to be tested in the clinic (9), Rexin-G has achieved regulatory approval in the Philippines for the treatment of all chemoresistant solid tumors, and is approaching regulatory approval in the USA for pancreatic cancer, osteosarcoma, and soft tissue sarcomas, where it has been granted FDA Fast Track designation and Orphan Drug status, respectively.

The clinical performance of Rexin- $\mathrm{G}$ is a function of the multiple levels of safety and efficacy embodied in its design engineering (1), as is its broad-spectrum anti-cancer activity (10). In terms of safety: i) the stealth vector platform allows repeated infusions without untoward immune responses; ii) the limitations of the retroviral platform become a virtue, as the vector is capable of enforcing gene expression in proliferative/dividing cells only; iii) the growth-associated designer gene is active against cancer cells and proliferative vasculature but not against normal non-dividing cells; and iv) the pathotropic accumulation in cancerous tissues essentially sequesters the vector away from non-target organs. In terms of efficacy: i) the cell cycle gene knockout provides for broadspectrum anti-cancer activity; while ii) the anti-angiogenic activity destroys tumor-associated vasculature; and iii) the pathotropic targeting leads to accumulation and high local concentrations where it is needed most, i.e., in the tumor microenvironment.

In this communication, we provide a compelling glimpse of pathotropic targeting in action: that is, the unique ability of Rexin-G nanoparticles to seek-out, accumulate in, and selectively deliver the 'killer gene' to metastatic liver lesions in a stage IVb pancreatic cancer patient, in the days preceding surgical excision of the residual tumor nodule and subsequent clinical remission of the disease. Alterations in the cellular biology of the aggressive metastatic cancer, as reflected in the observed histology, reveal the molecular mechanisms-of-action which rendered this residual tumor nodule amenable to surgical intervention.

\section{Case study and Methods}

The case study patient was a participant of an advanced phase I/II study employing a modification of the standard Cohort of Three design. A phase II efficacy component was incorporated in the phase I/II study by allowing additional treatment cycles to be given if the patient had equal or less 
than grade I toxicity. The principal investigator was also allowed to recommend surgical resection/debulking, and Rexin-G was continued if residual disease was found by histological examination or PET-CT scan (11).

An amendment to the New Investigational Drug application was approved by the FDA (BB-IND\#11586) in July, 2007, and the clinical protocol (C07-105) was reviewed and approved by the Western Institutional Review Board, Olympia, WA 98502. The phase I/II clinical trial using Rexin-G for pancreatic cancer was registered on www. clinicaltrials.gov (NCT00504998) within one week of study initiation, and patients were recruited on a first-come firstserved basis after appropriate screening procedures were conducted. Written informed consent was obtained from each patient at the time of enrollment.

Treatment. Rexin-G is a non-replicative 'pathotropic' or disease-seeking nanoparticle bearing a functional collagenbinding motif on its envelope protein and encoding an $\mathrm{N}$-terminal deletion mutant construct of human cyclin G1 under the control of a hybrid Moloney murine leukemia virus long terminal repeat promoter. The gene expression vector also contains the neomycin phosphotransferase gene driven by the SV40 early promoter, which is used for vector potency determinations. The Rexin-G vector is produced by transient co-transfection of three separate plasmids in 293T cells (human kidney 293 cells transformed with the SV40 large $\mathrm{T}$ antigen) maintained as a fully validated master cell bank. The final product exhibits a vector titer of $5 \times 10^{9}$ colony forming units (cfu) per milliliter, a biologic potency of $50-70 \%$ growth inhibitory activity in target cancer cells, $<1 \mathrm{~kb}$ residual DNA, no detectable E1A or SV40 large T antigen, and no detectable replication competent retrovirus (RCR). The clinical vector is stored in volumes of $23 \mathrm{ml}$ in $30 \mathrm{ml}$ glass vials at $-75 \pm 10^{\circ} \mathrm{C}$. Preparation of the Rexin-G vector for patient administration consisted of rapid thawing of the vector in the vial in a $34^{\circ} \mathrm{C}$ water bath. The vector was thawed within 15-30 min prior to infusion into the patient, and given intravenously at a rate (slow push) of $\sim 4 \mathrm{ml} / \mathrm{min}$. All personnel who handled and disposed of the retrovector observed Biosafety Level 2 compliance procedures in accordance with the National Institutes of Health Guidelines for Research Involving Recombinant DNA molecules.

The patient was given 4-treatment cycles, each cycle consisting of 4 weeks of intravenous infusions of Rexin-G, $4 \times 10^{11}$ cfu three times a week, followed by a two-week rest period. Surgical excision of the residual metastatic lesion in the liver was conducted after the 4 th cycle was completed, and adjuvant therapy with Rexin-G was given three weeks after surgery for five more treatment cycles.

\section{Results and Discussion}

This landmark case of metastatic pancreatic cancer is typical of many in which early detection and localization of disease enable a highly-beneficial surgical operation, referred to as a Whipple's procedure, wherein the primary tumor is removed with a partial resection of the pancreas. Unfortunately, disease recurrence is all too frequent, manifesting 'metastatically' in the liver, as in this case, with a predictably poor prognosis which generally precludes any further surgical intervention. More unfortunately, the results of standard first-line chemotherapy are measured in a few months, at best, and there is currently no evidence-based second-line therapy for pancreas cancer, though numerous chemotherapeutics and biologics have been tried (12-14). It is in this context that Rexin-G has out-performed all previous treatments (11) and now 'stands alone' as second-line therapy with FDA Fast Track status. Remarkably, this cancer patient was the last patient enrolled in an advanced and adaptive USA phase I/II study, which included a phase II efficacy component-allowing for continued Rexin-G infusions, provided as maintenance therapy in the absence of any systemic or dose-limiting toxicity. Routine follow-up studies affirmed overall safety, with no evidence of replication competency, vector integration in non-target organs, nor vector-neutralizing antibodies in patient samples.

While pancreatic cancer grows rapidly and aggressively, once it has invaded the parenchyma of the liver, often growing from one or two lesions to 'too-many-to-count' in a matter of months; this particular patient exhibited no new lesions during six months of continued Rexin-G treatment. Moreover, most of the metastatic liver lesions disappeared by CT scan, leaving a single life-threatening target lesion which had increased 2-fold in size. Remarkably, the tumor board at one notable Comprehensive Cancer Center in Los Angeles recommended a return to chemotherapy; however, a second opinion from another Los Angeles Medical Center reasoned that Rexin-G had controlled disease progression to the extent needed to consider surgical resection of the residual solitary tumor. In preparation for the surgery, Rexin-G was administered by intravenous infusion daily for three days according to the FDA-approved clinical protocol. The resulting surgical specimen was embedded for histological examination, as shown in Figs. 1-3.

The first plate (Fig. 1) is a composite revealing a largely fibrotic mass, with extracellular matrix occupying a large proportion of the tumor volume. The epithelioid tumor cells, identified by characteristic cytokeratins, are formed in arrays that appear like 'ductal' structures in various stages of formation and degeneration with appreciable focal necrosis. Notably, a significant amount of apoptosis (50\%) was observed within the tumor mass, a direct result of Rexin-G bioactivity $(5,6,15)$. Among the classic hallmarks of Rexin-G action-apoptosis, focal necrosis, anti-angiogenesis, immune cell infiltration, and reparative fibrosis, the patient's immune infiltrate is far from robust, but discernable as $\mathrm{CD}^{+}$leukocytes, helper-T and killer-T cells within the tumorous stroma, the latter of which tend to portend an improved overall survival.

The temporal proximity of the Rexin-G infusions to the surgical procedure encouraged an examination of the physical accumulation of Rexin-G nanoparticles within the tumorous tissues. Using a specific antibody directed against the targeted envelope protein of the Rexin- $G$ vector, immunohistochemical staining revealed appreciable accumulation of the nanoparticles and/or residual envelope proteins throughout the tumor nodule (Fig. 2). Reaching this tumor through the systemic circulation, the targeted nanoparticles had 

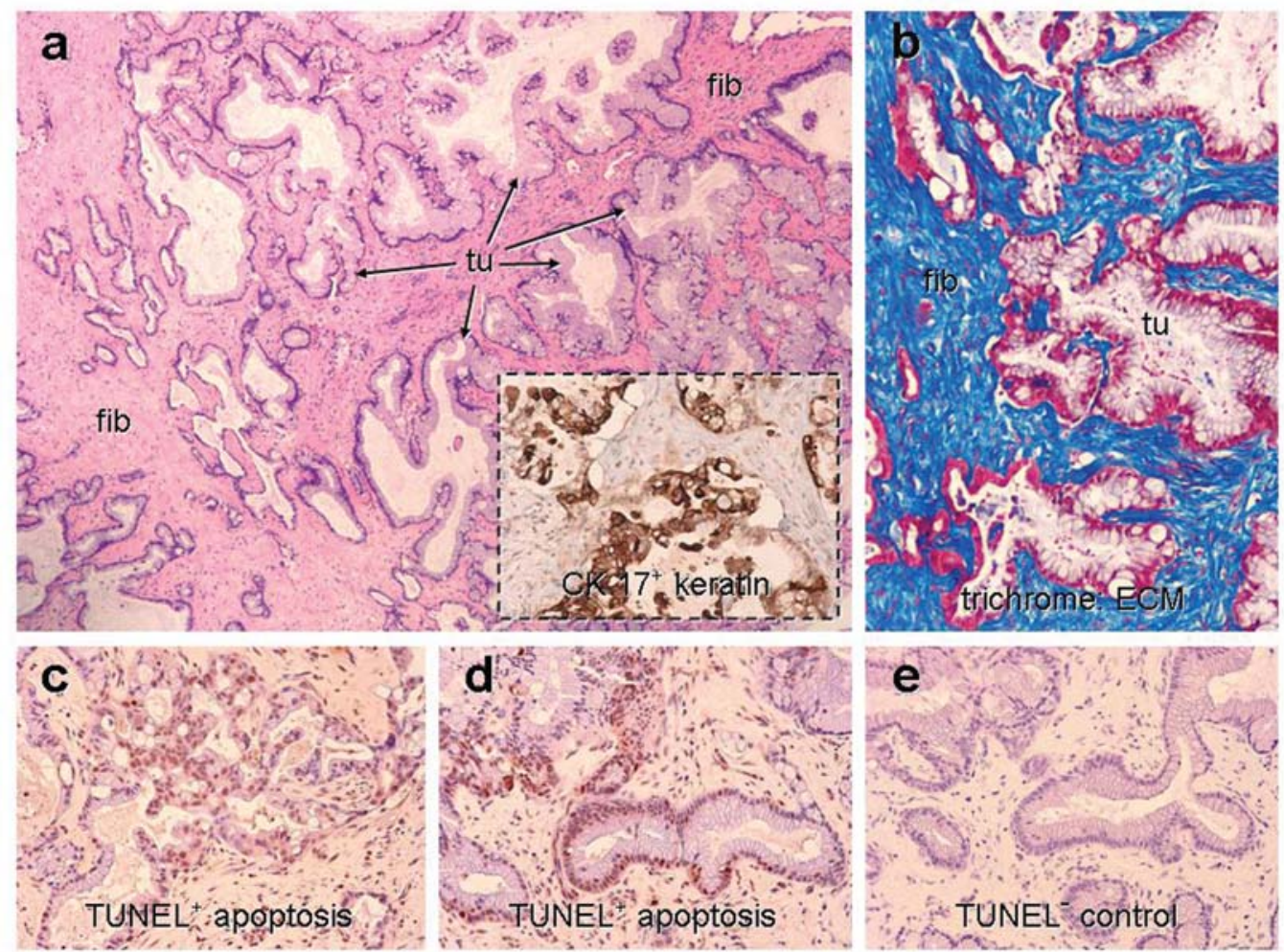

Figure 1. Histological section of excised liver tumor showing a preponderance of fibrosis (fib) with pseudo-differentiated epithelioid tumor cells (tu) arrayed in columnar/ductal structures, seen in various stages of degeneration (a, H\&E stain), as marked by a cytokeratin-17 immunostain (inset). Abundant fibrosis is observed throughout the tumor nodule, as shown by Masson's trichrome stain for ECM (blue stain, b). Remarkably, Rexin-G appears to have induced massive amounts of apoptosis of the pancreatic cancer cells (see TUNEL stain in c, d, and negative control e), as well as visible karyorrhexis and fragmentation, which is evident all along the borders of the pseudo-differentiated structures.
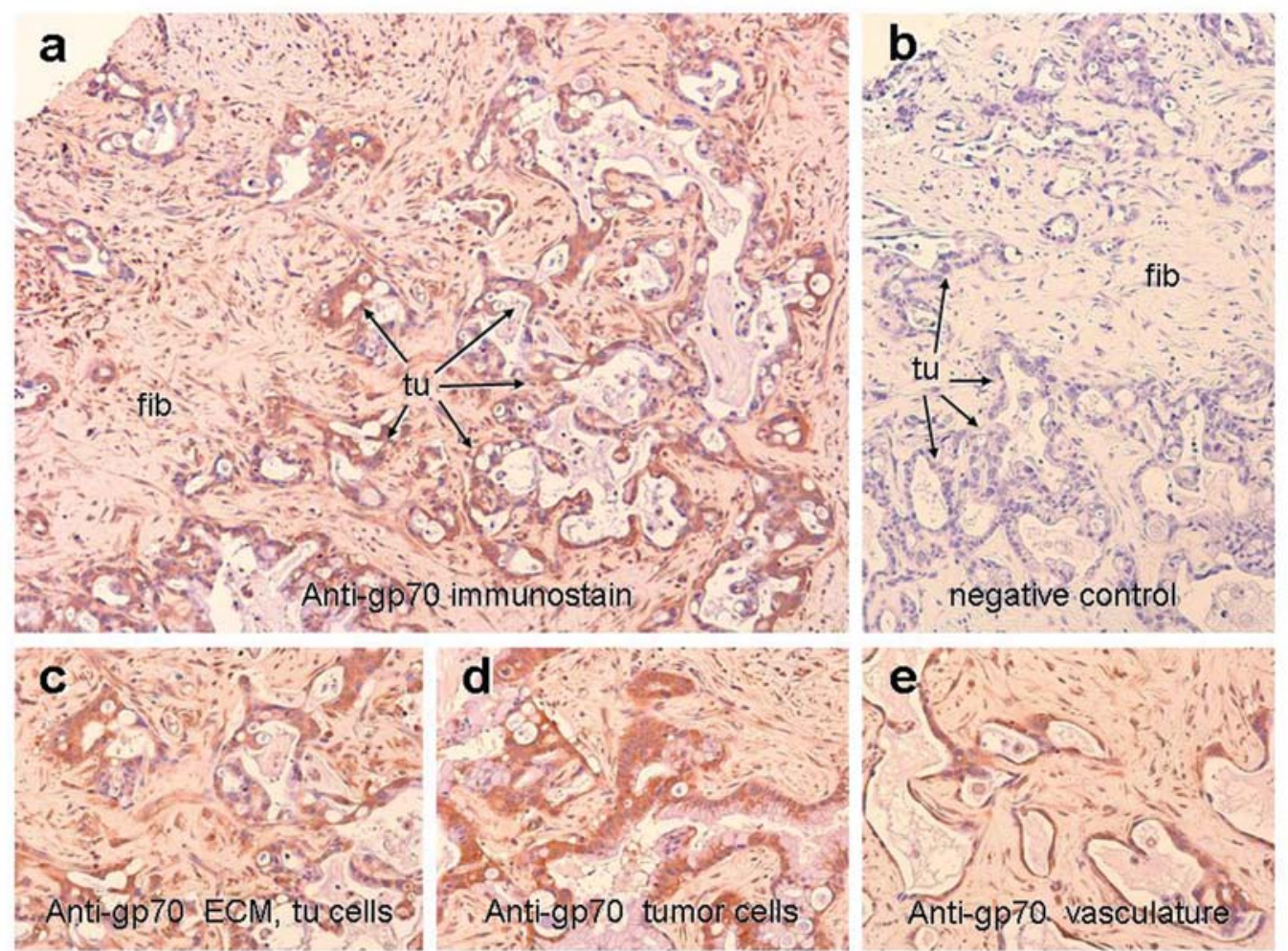

Figure 2. Immunohistochemical staining of the excised tumor for the gp70 envelope protein of the Rexin-G nanoparticle reveals an accumulation of immunoreactivity throughout the ECM-rich mass of the tumor (a vs. b, negative control), particularly in the cellular components, including the diffuse islands (c) and ductal structures (d) comprised of cancer cells and the elongate endothelial cells lining the vessels of the tumor-associated vasculature (e). 

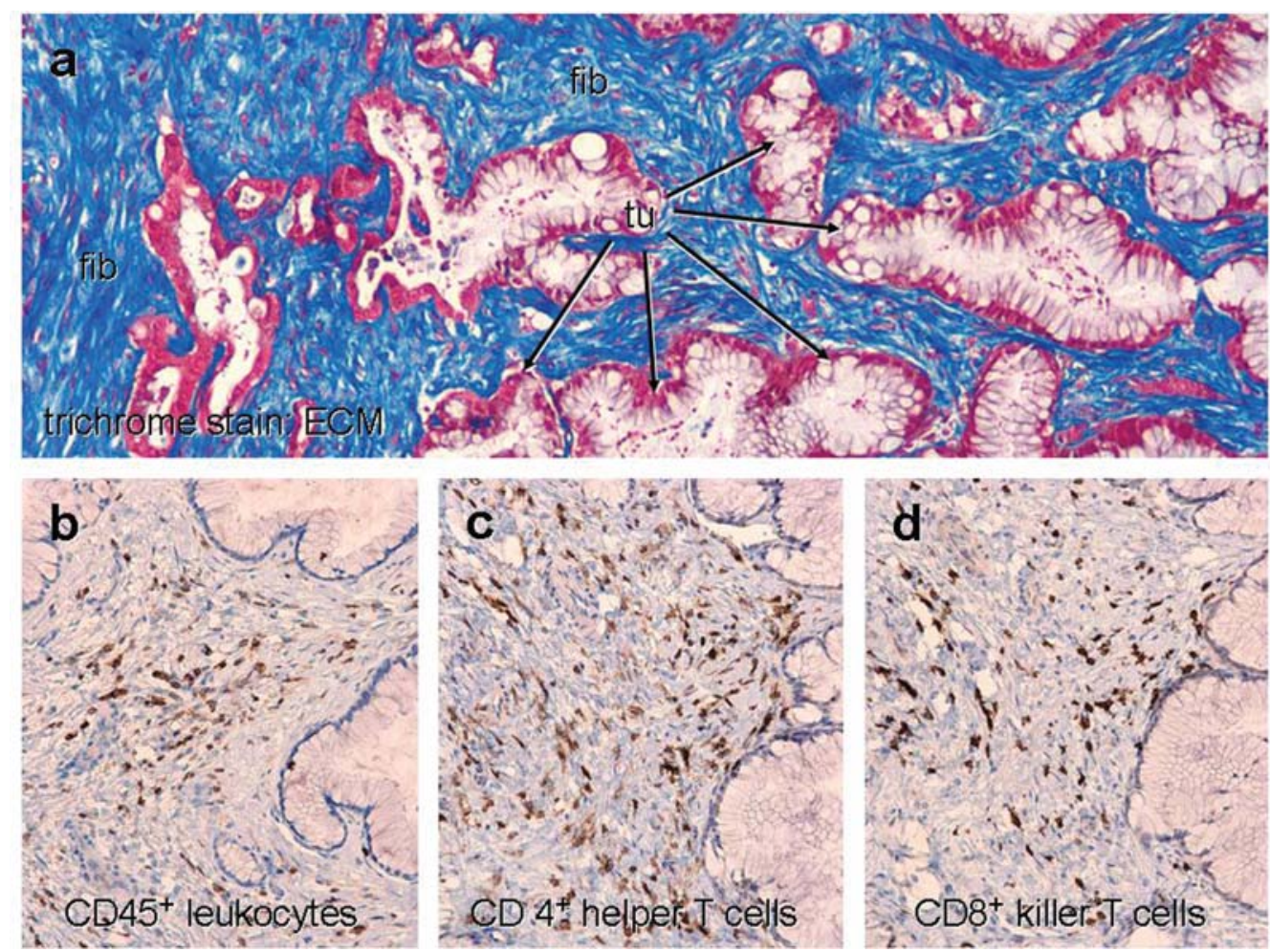

Figure 3. Further characterization of the immune infiltrate interspersed within the reactive/reparative fibrosis (fib) that surrounds the tumor cells (tu) of the excised nodule (a, Masson's trichrome stain, red for keratin, blue for ECM) reveals that the cadre of recruited immune cells, collectively marked by the CD45 common leukocyte antigen (b), contains both $\mathrm{CD}^{+}$helper T-cells (c) and CD8 ${ }^{+}$killer T-cells (d), the latter of which are selectively cytotoxic, adaptive components of cell-mediated tumor immunity.

transited the tumor vasculature and spread throughout the mass, much like dye particles in a natural sponge. While the biological half-life of the tumor-targeted nanoparticles is relatively short (measured in minutes), the localized accumulation of the telltale envelope protein is evident in areas of both fibrosis and tumor formation, with particular affinities for certain cellular components-most notably, tumor cells and endothelial cells of the associated neovasculature. This preferential affinity for these proliferative target cells presumably reflects the natural unimpaired affinity of this retrovector platform for its cellular receptor, the Pit2 sodiumdependent phosphate transporter (16), while the collagenor tumor-targeting property represents an auxiliary gainof-function (8). Taken together with the observation of extensive apoptosis of these target cells, this surgical biopsy serves to underscore the importance of collagen patefacio (i.e., collagen exposure) (10) as an important aspect and Achilles' heel of tumor formation, one that exposes the delicate and determinate fabric of nature, and which has now been utilized to therapeutic advantage.

With mindful attention to the clinical safety and efficacy of tumor-targeted Rexin-G $(10,15)$, and the gradual escalation of dosages to these highly effective levels, the implementation of pathotropic targeting may indeed change the manner in which cancer therapy will be administered and even evaluated in the future. As our understanding of the optimal dosing protocols, the precise mechanisms-of-action, and the extraordinary single-agent efficacy of Rexin- $G$ is further documented, this progress paves the way for surgical and medical oncologists to bring forth even greater patient benefits, in accordance with the evolving praxis of molecular medicine.

\section{References}

1. Gordon EM, Levy JP, Reed RA, Petchpud WN, Liu L, Wendler CB and Hall FL: Targeting metastatic cancer from the inside: a new generation of targeted gene delivery vectors enables personalized cancer vaccination in situ. Int J Oncol 33: 665-675, 2008

2. Gordon EM and Hall FL: A primer on pathotropic medicine. In: Celebrating One Hundred Years of the Food and Drug Administration. A special centennial edition. Brooklands New Media Ltd., Oswestry, Shropshire, UK, pp80-83, 2007.

3. Xu F, Prescott MF, Liu PX, Chen ZH, Liau G, Gordon EM and Hall FL: Long term inhibition of neointima formation in balloon-injured rat arteries by intraluminal instillation of a matrix-targeted retroviral vector bearing a cytocidal mutant cyclin G1 construct. Int J Mol Med 8: 19-30, 2001.

4. Gordon EM, Zhu NL, Prescott MF, Chen ZH, Anderson WH and Hall FL: Lesion-targeted injectable vectors for vascular restenosis. Hum Gene Ther 12: 1277-1287, 2001.

5. Gordon EM, Liu PX, Zhen SH, Liu L, Whitley MD, Gee C, Groshen S, Hinton DR, Beart RW and Hall FL: Inhibition of metastatic tumor growth in nude mice by portal vein infusions of matrix-targeted retroviral vectors bearing a cytocidal cyclin G1 construct. Cancer Res 60: 3343-3347, 2000.

6. Gordon EM, Liu PX, Chen ZH, Liu L, Whitley MD, Liu L, Wei D, Groshen S, Hinton DR, Beart RW, Anderson WF and Hall FL: Systemic administration of a matrix-targeted retroviral vector is efficacious for cancer gene therapy in mice. Hum Gene Ther 12: 193-204, 2001.

7. Gordon EM, Lopez FF, Cornelio GH, Lorenzo CC III, Levy JP, Reed RA, Liu L, Bruckner HW and Hall FL: Pathotropic nanoparticles for cancer gene therapy. Rexin-GTM: threeyear clinical experience. Int J Oncol 29: 1053-1064, 2006. 
8. Hall FL, Liu L, Zhu NL, Stapfer M, Anderson WF, Beart RW and Gordon EM: Molecular engineering of matrix-targeted retroviral vectors incorporating a surveillance function inherent in von Willebrand factor. Hum Gene Ther 11: 983-993, 2000.

9. Waehler R, Russell SJ and Curiel DT: Engineering targeted viral vectors for gene therapy. Nat Rev Genet 8: 573-587, 2007.

10. Gordon EM and Hall FL: The 'timely development' of Rexin-G: first targeted injectable gene vector (Review). Int J Oncol 35: 229-238, 2009

11. Nieto J, Grossbard ML and Kozuch P: Metastatic pancreatic cancer 2008: is the glass less empty? Oncologist 13: 562-576, 2008.

12. Burris H III and Rocha-Lima C: New therapeutic directions for advanced pancreatic cancer: targeting the epidermal growth factor and vascular endothelial growth factor pathways. Oncologist 3: 289-298, 2008.

13. Almhanna K and Kim R: Second-line therapy for gemcitabinerefractory pancreatic cancer: is there a standard? Oncology 22 $1176-1183,2008$.
14. Chawla SP, Chua VS, Fernandez L, Quon D, Blackwelder WC, Gordon EM and Hall FL: Advanced phase I/II studies of targeted gene delivery in vivo: intravenous Rexin-G for gemcitabine-resistant metastatic pancreatic cancer. Mol Ther 18: 435-441, 2009.

15. Gordon EM, Chan MT, Geraldino N, Lopez FF, Cornelio GH, Lorenzo CC III, Levy JP, Reed RA, Liu L and Hall FL: Le morte du tumour: histological features of tumor destruction in chemo-resistant cancers following intravenous infusions of pathotropic nanoparticles bearing therapeutic genes. Int J Oncol 30: 1297-1307, 2007.

16. Jobbagy Z, Garfield S, Baptiste L, Eiden MV and Anderson WB: Subcellular redistribution of Pit-2 P(i) transporter/amphotropic leukemia virus $(\mathrm{A}-\mathrm{MuLV})$ receptor in A-MuLV-infected NIH 3T3 fibroblasts: involvement in superinfection interference. J Virol 74: 2847-2854, 2000. 